\title{
Implementing psychotherapy guidelines: putting a logbook into practice
}

\author{
Chris Maloney
}

The integration of medical/'biologial' and psychological approaches to the human mind is unique to psychiatric training. There have been past tensions between the two views, but the ability to integrate them is increasingly recognised as an indispensable contribution of our profession to mental health care as a whole.

The College published 'Guidelines for psychotherapy training as a part of general professional training' in 1993 (Royal College of Psychiatrists, 1993). The authors emphasise the importance of an understanding of non-verbal communication, the complexities of the therapeutic relationship and the patient's internal world. This understanding should inform both specialist psychotherapeutic approaches, and psychiatric practice as a whole. Specific teaching methods are recommended: interview skills training and doctor-patient relationship seminars provide the basis for learning in the first year. Subsequently trainees should undertake supervised practice: a range of areas of experience is specified (see Table 1). Other issues worthy of attention, such as occupational stress, team working, and the integration of psychiatric and psychotherapeutic approaches are also identified.

Four years after the Guidelines appeared, information on their implementation is patchy: published surveys show major deficits even on prestigious training rotations, particularly in the areas of cognitive-behavioural therapy (CBT) and family therapy (Hamilton \& Tracy, 1996; Hwang \& Drummond, 1996; Lawson, 1996). Experience is valued, however, when it is received (Drummond \& Ramsay 1996: Lawson, 1996). In general. though specific modalities (CBT, medium-term psychodynamic) may be relatively well provided for, there seems to be widespread underprovision of the range and depth of recommended training (Hamilton \& Tracy, 1996; Hwang \& Drummond, 1996; Lawson, 1996).

\section{The need for psychological understanding}

For all we may be mocked or caricatured in popular culture, psychiatrists are still widely seen as specialists in human nature, able to manage difficult situations, and deal with disturbance in a creative and authoritative way. We cannot expect to do this by instinct and charisma alone, if we are not to disappoint those who put their trust in us.

Psychiatrists thus need training in psychological understanding and treatment. It is not sufficient to assume we pick up complex skills simply through socialisation into the profession. a sophisticated 'bedside manner'. We deal with psychologically damaged and distressed individuals who often react unusually in relationships - all psychiatrists need specific and rigorous skills in managing relationships with such people to their best advantage. Some may also work psychologically in more depth with patients, either within general psychiatric practice or as specialists. General professional

Table 1. Guidelines for psychotherapy training as part of general professional psychiatric training

\section{Clinical}

Experience

Individual

dynamic

psychotherapy

Individual

cognitive

behavioural-

therapy

Group therapy

One long-term case

Several time limited cases (e.g. 10 sessions)

One intensive case Several time-limited cases (e.g. 10 sessions)

In in-patient or out-patient settings, with experienced co-therapist or supervision

Marital and family Usually in child and family setting

therapy Includes psychosexual; therapy

Other experience Variety of setting Pathological bereavement Somatisation disorder Post-traumatic stress disorder Special techniques (e.g. psychodrama, art therapy, music therapy) 
training should provide a basis for developing these skills.

The Guidelines recognise these needs. Although perceived in some quarters as promoting the interests of a rather 'precious' subspeciality, at the expense of psychiatric training as a whole, this is not the case. Much of the recommended experience will only be found within general psychiatric placements: psychological understanding and skills integral to general psychiatry are recognised and valued.

\section{Types of psychotherapy}

Although in the past 'psychotherapy' may have connoted relatively inaccessible specialised psychodynamic services, the Guidelines use the term to indicate a range of psychological treatments. This usage is continued in the National Health Service Executive Report NHS Psychotherapy Services in England - A Review of Strategic Policy (Parry \& Richardson, 1996). Here the authors delineate three types of psychotherapy as a framework for understanding how services are delivered:

(a) Type A: psychological treatment as an integral component of mental health care

(b) Type B: eclectic psychological therapy and counselling

(c) Type C: formal psychotherapy.

This framework clarifies the aim of the Guidelines. It is not the intention to make all psychiatrists into practitioners of type $C$ therapy but instead to provide training in type $A$ and $B$ work for general psychiatric practitioners. The experience also serves as an introduction for those who then undertake a specialist training. This is not to say that the training should not be rigorous, it is not the degree of rigour that differentiates the three types.

From this perspective, it is possible to be clearer about how the Guidelines could be fulfilled. Psychotherapy training, in this sense, is not the preserve of specialist psychotherapy departments, who rarely have the resources to meet all the requirements; nor is it appropriate they do so. Both philosophically and practically, the necessary experience (including teaching and supervision) must be integrated throughout the training. This is only achievable as part of the daily work, and it needs to be a collaborative exercise between all those involved in training.

\section{Implementation in practice}

In the Oxford Deanery Training Scheme we have been reviewing how well we fulfil the Guidelines. A small survey revealed that, in keeping with the published work, we only partially meet the requirements. Best provision is in the three areas of Balint-type seminars, formal psychodynamic. and formal cognitive-behavioural work.

In 1996 a logbook was introduced throughout the training scheme. This is loose-leaf, with separate sheets for each recommended type of experience, requiring a brief description of the work, and the supervisor's signature. In the absence of clear procedures for its use, few trainees were completing it. A working party was thus formed early in 1997 to examine how the psychotherapy training relates to general professional training as a whole.

Our findings are presented in the hope of showing practical ways of addressing the issues that may be of use to others in developing this central aspect of psychiatric training.

\section{Recommendations of the Oxford Deanery Working Party}

Provision of psychotherapy experience for trainee psychiatrists has to be a collaborative exercise across their training. The logbook should help trainees to structure their psychotherapy experience, and provide a basis for petitioning for particular experience if not available. Certain key individuals can help in this.

\section{Provision of logbook}

College tutors supply the logbook to new trainees at induction or shortly afterwards. Those who have already done some psychiatry are given the logbook to fill in retrospectively. The logbook is contained in a loose-leaf A4 binder, the 'Record of Training'. This also has dividers to accommodate briefing and appraisal forms, a copy of the psychotherapy guidelines, and the trainees' ongoing curriculum vitae. This training record is taken to new placements as their rotation proceeds, forming the basis of the 'briefing session' with each new educational supervisor, when educational objectives for the placement are set.

\section{Supervision and availability of experience}

A 'supervisor' is any mental health professional skilled in the particular type of treatment they are supervising. 'Supervision' is consistent, regular meetings to discuss the work in detail. This formal contract is essential to the experience, satisfaction with clinical supervision has been shown to be far greater for supervisees with a formal contract, regardless of model (Arnott et al, 1996).

For some experience, such as long-term group work, it may not be practical for the trainee to act as an ongoing therapist. Other experience, perhaps as an observer, or participating in administrative arrangements, is valuable and 
should be recorded, making it explicit what was involved.

\section{Achieving the psychotherapy training}

The 'trainee' is ultimately responsible for obtaining the required experience and entering it into the logbook. Five key individuals help with this.

The educational supervisor reviews the logbook during the briefing session in the first two weeks of the job. Experience available in the placement can be planned (e.g. group work on the ward, cognitive work, couple work etc.). This is part of the educational plan for the job, and its achievement is reviewed at the end of placement appraisal.

Specialist consultant psychotherapist and/or department: the consultant psychotherapists oversee provision of formal specialist therapies for their district (e.g. psychodynamic, cognitivebehavioural), and provide supervision as appropriate. A local psychotherapy department may not be directly able to provide experience in all modalities (commonly, for example, CBT is provided by psychologists), but is well placed to facilitate the trainee getting the necessary experience. In cases of difficulty this may be pursued in collaboration with the college tutor.

The college tutor supports the trainee in obtaining experience and facilitating opportunities in the scheme in general. The psychotherapy logbook is reviewed as an integral part of the annual appraisal. Gaps are identified, and plans made to fill them, to be reviewed in succeeding appraisals. Particular problems may be discussed with specialist psychotherapists in the district.

The scheme organiser is made aware of any particular difficulties after the annual appraisal: there is also liaison with the regional speciality tutor to discuss the overall level of psychotherapy experience available, and whether this is satisfactory.

\section{Audit of psychotherapy training within the scheme}

Retrospective surveys, usually with response rates of $50-70 \%$, do not provide adequate information about the range and comprehensiveness of available experience; more comprehensive, prospective information gathering is needed. College tutors complete a summary form for each trainee at the annual appraisal: a list of possible experience, with check boxes and details of length of training and posts held to date. This is submitted to the regional speciality tutor. This will provide 'normative data' as to the feasibility of fulfilling the guidelines, and identify specific gaps in available experience.

\section{Conclusions}

This process should facilitate psychotherapy training for the majority of interested and committed trainees. It may also identify those who are particularly non-participatory or disruptive, but this is not its main intention.

The Guidelines are important in fitting the long-standing requirements for formal psychotherapy experience into a broader picture. They identify valuable ways of teaching 'psychological mindedness' to psychiatrists. Some trainees take to this naturally, others are more reluctant. Healthy scepticism should be encouraged - some doctors may have difficulties with psychological concepts (particularly psychodynamic), but are prepared to think about them. and challenge them in a rigorous and constructive way.

The Guidelines are not about enforcing a particular world view or model of practice, but about ensuring that trainees can engage with different views and perspectives on their daily work. The range of experience and techniques encompassed is a testimony to this - hopefully every trainee will be exposed to at least one way of working with which they are uncomfortable, or with which they disagree. The aim of all good education is to provoke thought.

\section{Acknowledgements}

Many thanks to Drs John Baruch, Zubin Bhagwagar, Margaret Bransford, and Rebecca Mather, who sat with me on the working party.

\section{References}

ARNOTt, S. DAWkInS, E. \& AYLARD, P. (1996) 'The Sorcerer and the Apprentice". a study of clinical supervision of psychotherapy and counselling occurring in a districtwide general adult psychiatric service. Psychiatric Bulletin, 20, 609-611.

DRumMOND, L. M. \& RAMSAY, R. L. (1996) Trainees' experience and attitudes to behavioural-cognitive psychotherapy training. Psychiatric Bulletin. 20. 351-353.

HAMILTON, R. J. \& TRACY. D. (1996) A survey of psychotherapy training among psychiatric trainees. Psychiatric Bulletin, 20, 536-537.

HWANG, K. S. \& DRUMmond. L. M. (1996) Psychotherapy training and experience of successful candidates in the MRCPsych examinations. Psychiatric Bulletin. 20. 604 606.

LAWSON. L. (1996) College guidelines on psychotherapy training. Psychiatric Bulletin, 20. 695.

PARRY, G. \& Richardson, A. (1996) NHS Psychotherapy Services in England-A Review of Strategic Policy. London: NHS Executive.

Royal College of PSYchiatrists (1993) Guidelines for psychotherapy training as part of general professional psychiatric training. Psychiatric Bulletin, 17. 695-698.

Chris Maloney, Consultant Psychotherapist, Heatherwood Hospital, London Road, Ascot SL5 $8 A A$ 